\title{
Simulation of LTE-TDD in the HAPS channel
}

\author{
Aws Majeed Al_Awadi', Mohammed Jawad Al_Dujaili² \\ ${ }^{1}$ Communication and Media Commission Baghdad, Iraq \\ ${ }^{2}$ Department of Electronic and Communication, Faculty of Engineering, University of Kufa, Iraq
}

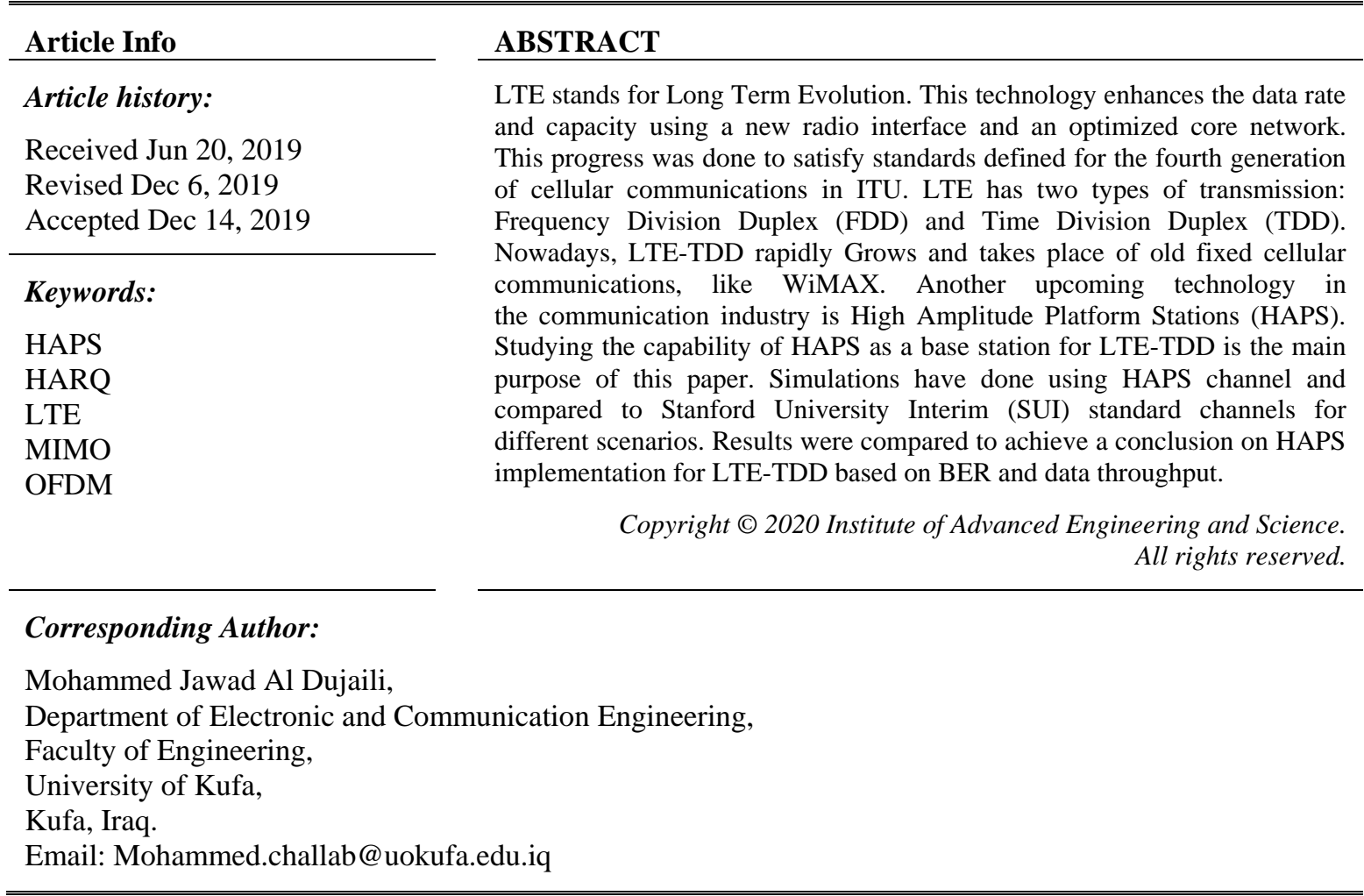

\section{INTRODUCTION}

Nowadays, cities are growing so fast and hence base stations should be replanted to maintain the network coverage. Network planning is a time-consuming process and costs a lot. The best solution is using a base station that can relocate easily, and its communication takes a minimum effect of multipath from buildings. Because of the high altitude of HAPS, it can provide a communication link similar to satellites. This link is resistant to horizontal multipath and makes HAPS a good alternative for fixed land base stations. Also, LTE is a leading technology in cellular communication with good sustainability against destructive effects in radio channels. To study the performance of LTE on HAPS, the different situation should be simulated. In [1] MIMO communication was analyzed for HAPS, but this article does not simulate physical layer. First effort for implementation of the broadband physical layer for HAPS was done for WiMAX in [2-4]. After presenting LTE technology researchers started to study its implementation on HAPS [5-7]. The main simulation in articles studies FDD type of LTE till today. Because of the good performance of TDD-LTE in fixed cellular networks, this paper is going to simulate this type of LTE for HAPS. In the next section LTE technology is introduced. The third section consists of HAPS introduction and its channel simulation methods. In the fourth section a brief introduction of MIMO is presented. In the fifth section, the simulation scenario is explained. In the sixth section results with complete discussion is presented. At the end conclusion is made on the results. 


\section{LTE}

Long Term Evolution (LTE) is a standard for wireless broadband communication. It increases the capacity and data rate by using a different radio interfaces together with core network improvements. In the radio interface, LTE uses Orthogonal Frequency Division Multiplexing (OFDM). This is a method of encoding digital data on multiple carrier frequencies without any frequency guard band. Also, unlike UMTS that uses fixed $5 \mathrm{MHz}$ bandwidth, LTE can utilize a various range of bandwidth from $1.4 \mathrm{MHz}$ to $20 \mathrm{MHz}$. This allows operators to flexibility to choose the desired configuration to depend on their dedicated spectrum and data traffic. LTE has two data transmission technologies, Frequency Division Duplex (FDD) and Time Division Duplex (TDD). While LTE-FDD uses paired frequencies to upload and download data, LTE-TDD uses a single frequency, alternating between uploading and downloading data through time. The ratio between uploads and downloads on an LTE-TDD network can be changed dynamically, depending on whether more data needs to be sent or received. Because of these advantages, nowadays LTE-TDD is used as a fixed cellular communication solution in many countries. This technology is the best choice for reaching a new era of communication $[8,9]$.

\section{HAPS}

High-Altitude Platform Station or High-Altitude Pseudo-Satellite (HAPS) is defined as "a station on an object at an altitude of 20 to $50 \mathrm{~km}$ and at a specified, nominal, fixed point relative to the Earth". This platform is able to deliver high-speed connectivity to users, over areas of up to $400 \mathrm{~km}$. It has gained significant interest because HAPS will be able to deliver bandwidth and capacity similar to a broadband wireless access network (such as WiMAX) while providing a coverage area similar to that of a satellite. It can provide Line of sight communication similar to GEO satellites, which guaranteed low latency and minimum path loss effects. Also, multiple HAPS platforms can be synchronized to form a cellular network and take advantage of frequency reuse. HAPS is more cost efficient than a communication satellite and is more flexible than a fixed land base station, with also a better coverage. Considering these benefits, HAPS is a future step in providing fixed cellular communication [10].

HAPS channel acts like satellite channel. This channel has a line of sight, so transmitted power attenuated proportionally to the square of the distance. Mostly, fading in satellite and HAPS channel describes using Ricean distribution function, but in dense areas like large cities, Rayleigh distribution function is preferred. There are four major channels modeling for HAPS:

- Practical model (described in[11-14])

- Geometrical model (described in[1, 15, 16])

- Statistical model (described in[2, 3])

- Multistate model (described in [4, 17-19])

Most of the presented channels for HAPS are modelled with the narrowband assumption. This means these models do not consider incoherent bandwidth for broadband communication. Because of time-variant nature in real channels, broadband channels model using Tapped Delay Line (TDL). In this model, each tap has different weights and distributions. Articles $[20,21]$ used a TDL model for simulating HAPS channel. This paper uses HAPS models presented in these articles.

\section{MIMO}

Multiple Input and Multiple Output (MIMO) is a method for multiplying the capacity of a radio link, using multiple transmissions and receiving antennas to exploit multipath propagation. Multipath propagation of signal is the main limiter for high rate data transmission. Received signal also suffers from other phenomena like fading, Co-Channel Interference (CCI), and Inter-Symbol Interference (ISI). When there isn't enough knowledge on the channel in the transmitter or channel that varies very fast, the best solution is diversity coding provided by MIMO. In diversity methods, a single stream (unlike multiple streams in spatial multiplexing) is transmitted, but the signal is coded using techniques called space-time coding. The signal is emitted from each of the transmit antennas with full or near orthogonal coding. Diversity coding exploits the independent fading in the multiple antenna links to enhance signal diversity. As Proposed by Vahid Tarokh, Nambi Seshadri, and Robert Calderbank, these space-time codes (STCs) achieve significant error rate improvements over single-antenna systems. Their original scheme was based on trellis codes but the simpler block codes were utilized by Siavash Alamouti to develop space-time block-codes (STBCs) [22]. In Alamouti coding, two consecutive symbols are coded coded in 2 by 2 orthogonal matrix. Each column sent from two MIMO antennas at each burst of transmission. In the receiver, signals gather from two MIMO antennas and combine to construct sent symbols. In this paper, Alamouti coding is implemented using 2 by 2 MIMO scheme. 


\section{SIMULATION SCENARIO}

The stimulated algorithm is shown in Figure 1. At the first step of algorithm random data produced. Then data are divided into two streams for each MIMO antenna. After that each data encoded. In the encoding step, first of all, Cyclic Redundancy Check (CRC) added to data stream as an error-detecting code, and then Reed Solomon codes added to data as Forward-Error-Correction (FEC) coding. At least data coded using Viterbi and then modulated with defined modulation order. Then in OFDM modulator block, data transformed to the time domain using Inverse Fast Fourier Transform (IFFT). After that guard band and a cyclic prefix (CP) added to modulated data. At this level, Altamonte block queues these symbols according to STBC and TDD scheme for transmission. Prepared data transmitted through time-varying HAPS channel. Parameters of this channel can be defined by the user. In this paper, just the downlink simulated with TDD uplink/downlink configuration type 1. It means half of the time date sent from HAPS toward a receiver on the ground, and at the rest of the time, ACK/NACK data sent back to the HAPS. At the receiver side, data catch using Altamonte receiver. This receiver combines the signal from two consecutive receives and also tries to omit channel effects from it. In the OFDM demodulator guard band and CP eliminated. After that signal bring back to the frequency domain using Fast Fourier Transform (FFT). In the decoder, block data extract using a Viterbi decoder and corrected using Reed Solomon codes. After that, CRC codes checked to see received data is valid or not. At this level, if we have no error in data ACK sent back to the transmitter to send the next data. If the data has an error, NACK sent back to the transmitter to inform it for sending the same data. After resending data HARQ combines two new received signals with the older one to increase SNR. This action repeated for 4 times if an error does not eliminate. The number of successive pack of received data compare to the total sent data stream for calculating throughput. At last the received data checked with raw data at the transmitter to calculate BER.

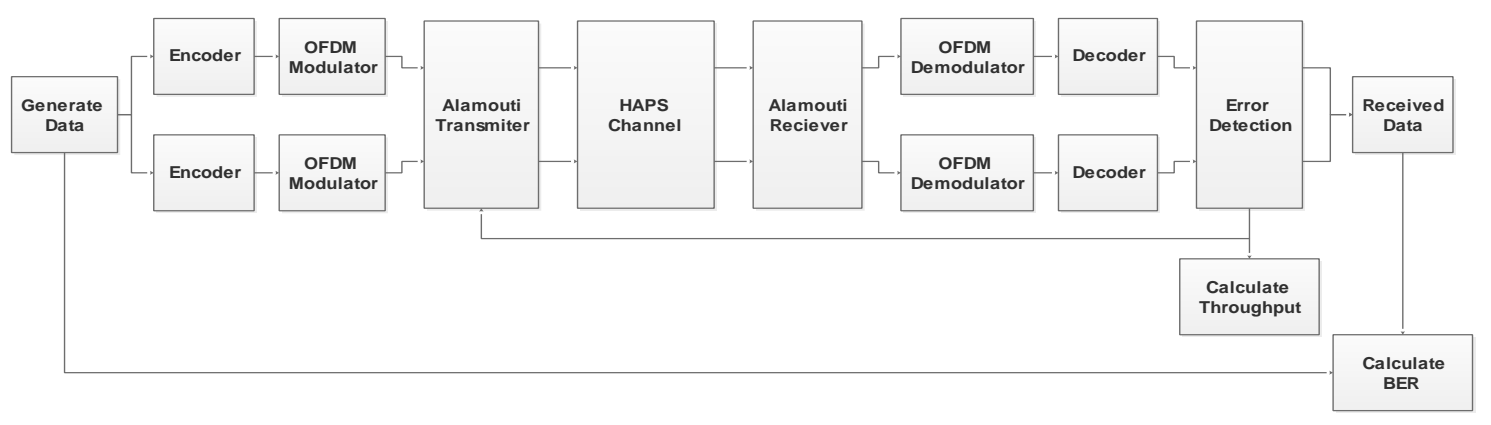

Figure 1. Stimulated algorithm

\section{SIMULATION RESULT}

First of all, it's better to find the best settings for comparing HAPS channel with SUI channels. Modulation is the first concern. So, simulation was done using $5 \mathrm{MHz}$ as the lowest commercial bandwidth, cycle prefix equal to 0.125 and various modulations. Figure 2 shows the result of throughput against SNR and Figure 3 shows a BER comparison against SNR.

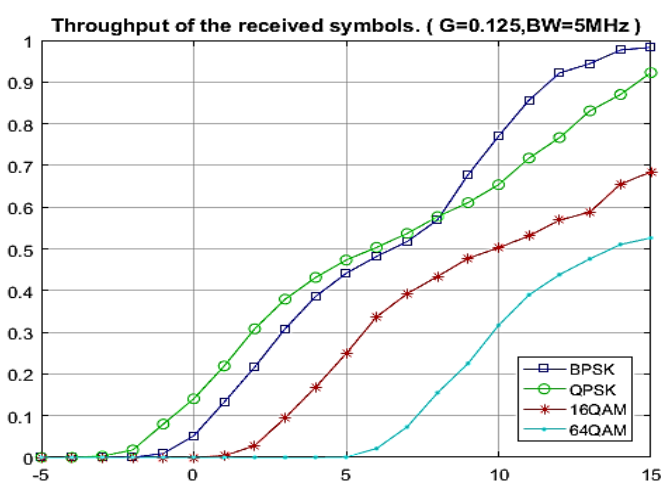

Figure 2. Throughput against SNR

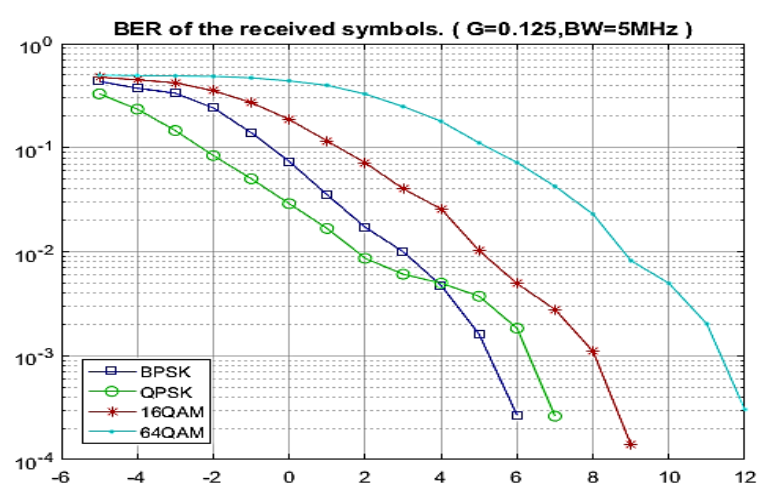

Figure 3. BER comparison against SNR 
As it can be seen in the low SNR QPSK has better performance than the other modulations. In the SNR rate higher than $4 \mathrm{~dB}$, BPSK shows a better result. Because of the high altitude of HAPS, it can be expected that the received signal has a low SNR. So QPSK modulation selected for the rest of simulations. Next simulation was done to choose the best cyclic prefix. Properties are set to $5 \mathrm{MHz}$ for bandwidth, QPSK as modulation and the CP is variable. Figure 4 shows the result of throughput against SNR and Figure 5 shows a BER comparison against SNR.

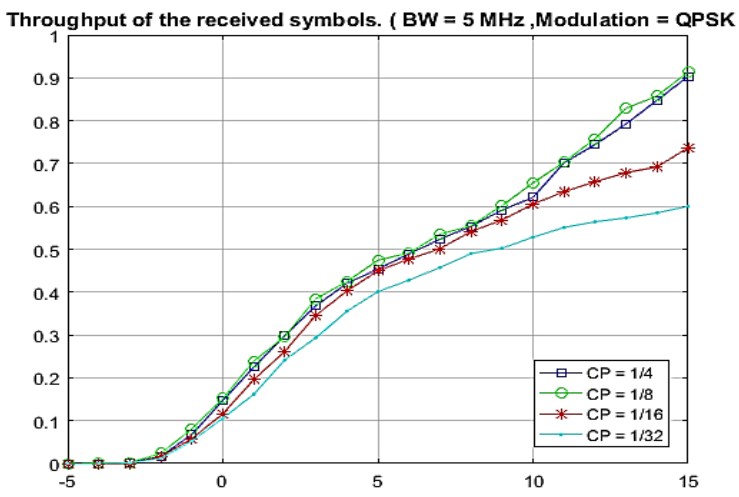

Figure 4. Throughput against SNR

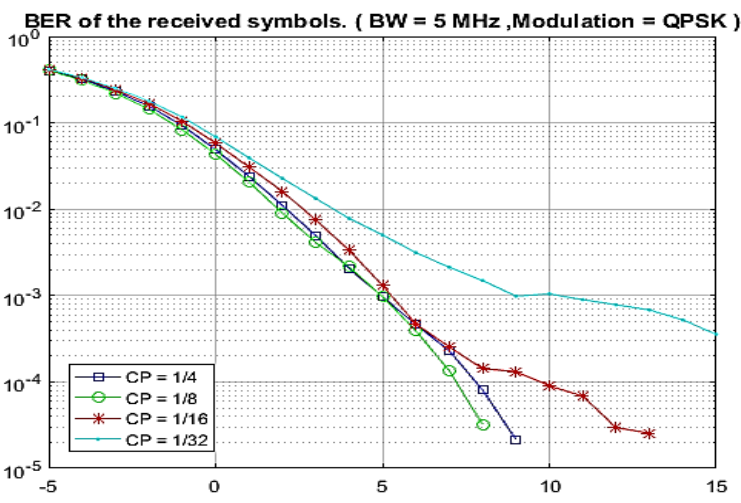

Figure 5. A BER comparison against SNR

As can be seen, $\mathrm{CP}$ of 0.25 has the best result, but higher $\mathrm{CP}$ means we should lose a great portion of time for sending redundant data. $\mathrm{CP}$ of 0.125 has the same outcome as a 0.25 , so we choose it as the main $\mathrm{CP}$ for the rest of the simulations. Next, simulation was done to evaluate bandwidth effects. In this simulation $\mathrm{CP}$ equal to 0.125 , QPSK as modulation and bandwidth is variable. Figure 6 shows the result of throughput against SNR and Figure 7 shows a BER comparison against SNR.

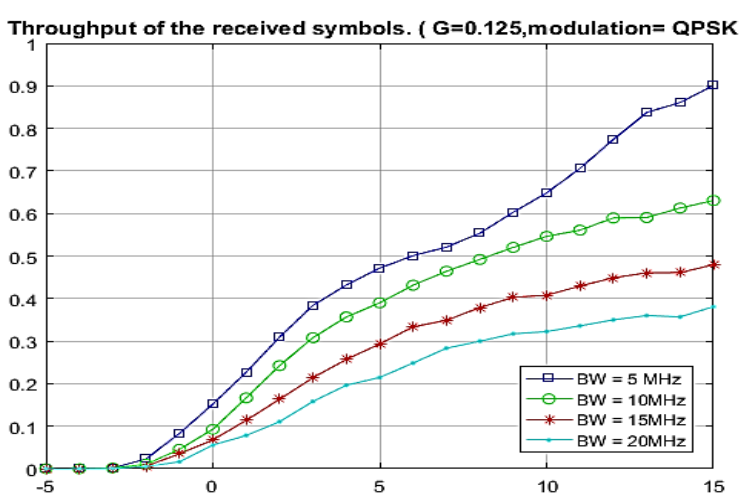

Figure 6. Throughput against SNR

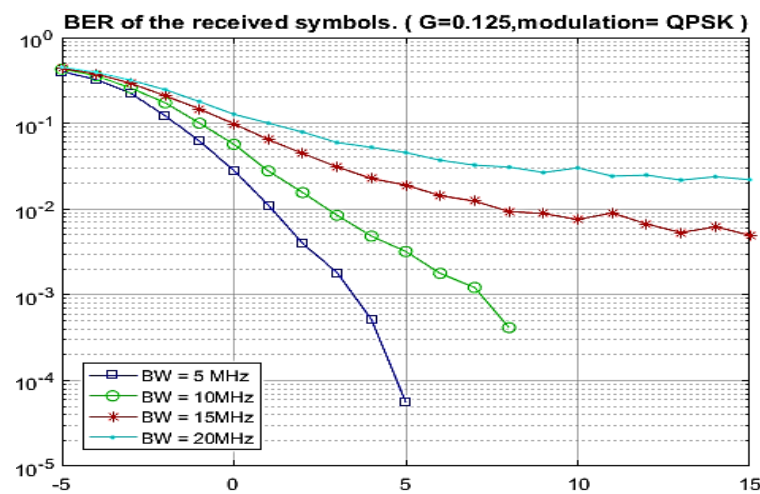

Figure 7. BER comparison against SNR

As it can be predicted, a lower bandwidth is more resistant to the noise of the channel. So, $5 \mathrm{MHz}$ of bandwidth is the best option for sending data in the HAPS channel. In the next step, by reaching to the best option, the simulation was done to compare the HAPS channel with SUI channels. SUI channels add verb completely in [23, 24]. SUI-1 \& SUI-2 defines mostly flat terrain with light tree densities. It corresponds to minimum path loss conditions comfort for suburban areas. SUI-2 has more delay spread than SUI-1. SUI-3 \& SUI-4 defines hilly environment but rare vegetation, or high vegetation but flat terrain. Intermediate path loss condition is typical of this category and can model urban area for small to medium cities. Again SUI-4 has more delay spread than SUI-3. SUI-5 \& SUI-6 defines hilly terrain with moderate-to-heavy tree densities, which results in the maximum path loss. It used to model metropolis with large buildings. SUI-6 has more delay spread than SUI-5. For these simulation properties set to the bandwidth of $5 \mathrm{MHz}$, the cyclic prefix of 0.125 and modulation of QPSK. Figure 8 shows the result for throughput against SNR and Figure 9 shows a BER comparison against SNR [25]. 


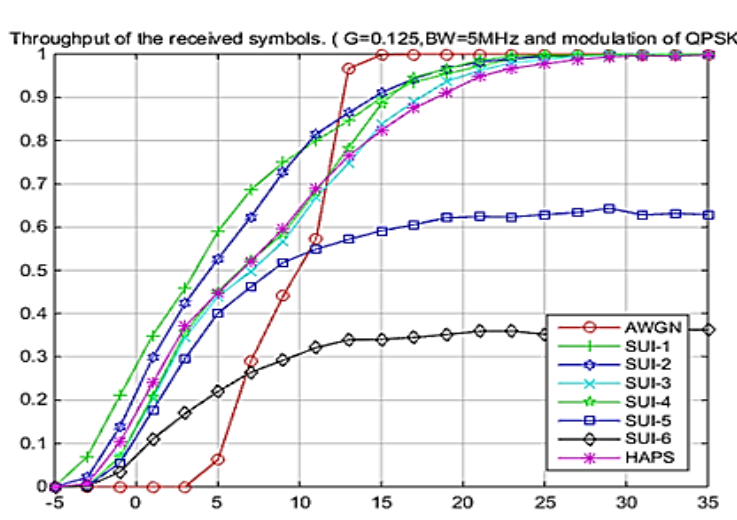

Figure 8. Throughput against SNR

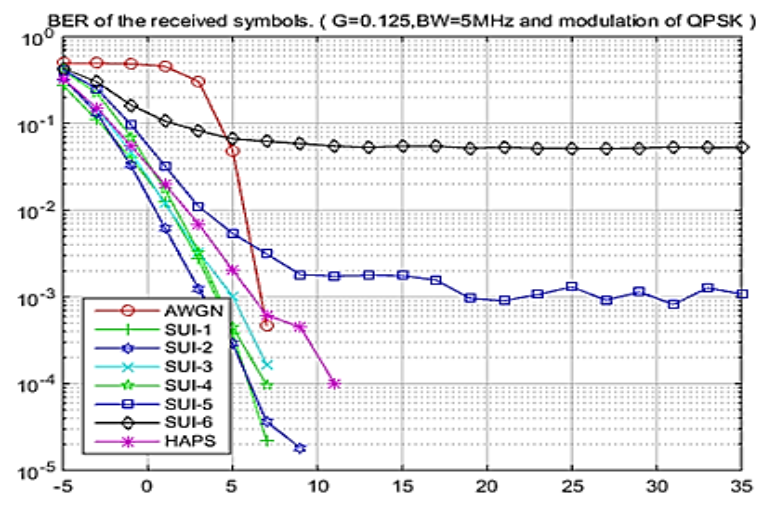

Figure 9. BER comparison against SNR

As it can be seen, the HAPS channel has a similar behavior as the SUI-3 \& SUI-4 and act away better than SUI-5 \& SUI-6. It means using HAPS for TDD-LTE in the urban area is a good decision. As it mentioned SUI-3 has low spreading delay \& SUI-4 has a medium spreading delay. HAPS has a better performance than SUI-4 under SNR rate equal to $0 \mathrm{~dB}$, and also has a better performance than SUI-4 under SNR rate equal to $-3 \mathrm{~dB}$. It shows that in the environment with high noise, or conditions that can receive enough power in the receiver point, HAPS has better performance for the urban area. In the suburban areas fixed land network act better than the HAPS depends on the result for SUI-1 \& SUI-2. At the next section, a more comprehensive conclusion takes for this comparison.

\section{CONCLUSION}

In the metropolis and hilly terrain with moderate-to-heavy tree densities, HAPS is the best choice for a cellular network. In the urban area with medium building densities, if the fixed land network can't provide enough power, HAPS has a better performance in the low SNR rate. Just in the flat area with low densities of trees and building like suburban, the fixed land network performs better than HAPS. However, in these regions, HAPS has other superiority. HAPS has coverage of about $400 \mathrm{~km}$, while maximum coverage of land base station is limited to $70 \mathrm{~km}$ in the ideal condition. So, it can be concluded that HAPS can successively supersede the fixed land cellular network for TDD-LTE technology.

\section{REFERENCES}

[1] E.T. Michailidis and A.G. Kanatas, "Statistical simulation modeling of 3-D HAP-MIMO channels," Wireless Personal Communications, vol. 65, no. 4, pp. 833-841, 2012.

[2] I.-R. Palma-Lazgare, J.-A. Delgado-Penin, F. Perez-Fontan, "An advance in wireless broadband communications based on a WiMAX-HAPS architecture," in 26th international communications satellite systems conference (ICSSC), 2008.

[3] E. T. Michailidis and A. G. Kanatas, "Three-dimensional HAP-MIMO channels: Modeling and analysis of spacetime correlation," IEEE Transactions on Vehicular Technology, vol. 59, no. 5, pp. 2232-2242, 2010.

[4] D. R. Putro, "Performance evaluation of broadband WiMAX services over high altitude platforms (HAPs) Communication Channel," ICWMC'08, The Fourth International Conference on Wireless and Mobile Communications, IEEE, pp. 55-59, 2008

[5] S. Gratsia and M. Ernawan, "LTE uplink cellular capacity analysis in a high altitude platforms (HAPs) communication," in 11th International Conference on Telecommunication Systems Services and Applications (TSSA), IEEE, pp. 1-5, 2017.

[6] M. R. K. Aziz, "Channel estimation for LTE downlink in high altitude platforms (HAPs) systems," in International Conference of Information and Communication Technology (ICoICT), IEEE, pp. 182-186, 2013.

[7] M. Aziz, "A study of HAPS-LTE downlink channel performance simulation deployed for high speed user vehicle," in 8th International Conference on Telecommunication Systems Services and Applications, IEEE, pp. 1-5, 2014.

[8] S. Sesia, M. Baker, and I. Toufik, "LTE-the UMTS long term evolution: from theory to practice," John Wiley \& Sons, 2011.

[9] B. Furht and S. A. Ahson, "Long Term Evolution: 3GPP LTE radio and cellular technology," Crc Press, 2016.

[10] X. Cao, P. Yang, M. Alzenad, X. Xi, D. Wu, and H. Yanikomeroglu, "Airborne communication networks: A survey," IEEE Journal on Selected Areas in Communications, vol. 36, no. 9, pp. 1907-1926, 2018.

[11] L. Hanzo, Y. (Jos) Akhtman, L. Wang, and M. Jiang, "MIMO-OFDM for LTE, Wi-Fi and WiMAX," ed: John Wiley and Sons Ltd., United Kingdom, 2011. 
[12] H. S. Wang and N. Moayeri, "Finite-state Markov channel-a useful model for radio communication channels," IEEE Transactions on Vehicular Technology, vol. 44, no. 1, pp. 163-171, 1995.

[13] J. L. Cuevas-Ruíz and J. A. Delgado-Penín, "Channel modeling and simulation in HAPS systems," In Proceedings of the Fifth European Wireless Conference, Spain, pp. 24-27, 2004.

[14] A. Imran and R. Tafazolli, "Performance \& capacity of mobile broadband WiMAX (802.16 e) deployed via high altitude platform," in Wireless Conference, IEEE, pp. 319-323, 2009.

[15] J. L. Cuevas-Ruiz and J. A. Delgado-Penín, "A statistical switched broadband channel model for HAPS links," in WCNC, pp. 290-294, 2004.

[16] I.R. Palma-Lázgare, J.A. Delgado-Penín, "WiMAX haps-based downlink performance employing geometrical and statistical propagation-channel characteristics," URSI Radio Science Bulletin, vol. 2010, no. 333, pp. 50-66, 2010.

[17] A. Ghosh, D. R. Wolter, J. G. Andrews, and R. Chen, "Broadband wireless access with WiMax/802.16: current performance benchmarks and future potential," IEEE Communications Magazine, vol. 43, no. 2, pp. 129-136, 2005.

[18] C. Hoymann, "Analysis and performance evaluation of the OFDM-based metropolitan area network IEEE 802.16," Computer Networks, vol. 49, no. 3, pp. 341-363, 2005.

[19] R. Miura and M. Oodo, "Wireless communications system using stratospheric platforms: R and D program on telecom and broadcasting system using high altitude platform stations," Journal of the Communication Research Laboratory, vol. 48, pp. 33-48, 2001.

[20] Z. Lian, L. Jiang, and C. He, "A 3-D wideband model based on dynamic evolution of scatterers for HAP-MIMO channel," IEEE Communications Letters, vol. 21, no. 3, pp. 684-687, 2017.

[21] Y. Ding, Y. Xiao, J. Xie, and T. Zhang, "A time-varying transition channel model for air-ground communication," IEEE/AIAA 36th Digital Avionics Systems Conference (DASC), pp. 1-8, 2017.

[22] S. Alamouti, P. Poon, and V. Tarokh, "Low complexity maximum likelihood detecting of concatenated space codes for wireless applications," ed: Google Patents, 2002.

[23] R. Jain, "Channel models: A tutorial," in WiMAX forum AATG, pp. 1-6, 2007.

[24] Cuevas-Ruíz, José Luis, and José A. Delgado-Penín. "Channel modeling and simulation in HAPS systems," European Wireless 2004, pp. 24-27, 2004.

[25] Jackson, Thomas, George Eapen, and Fa Dai, "Linearized offset QPSK modulation utilizing a sigma-delta based frequency modulator," U.S. Patent No. 6,975,687. 13 Dec. 2005.

\section{BIOGRAPHIES OF AUTHORS}

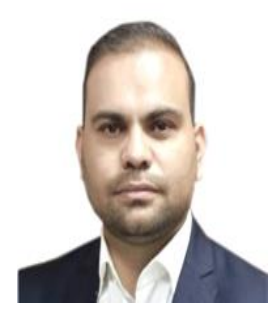

Aws Majeed Ghalib Alawadi, Associate Director of the communication Regulatory Authority, Head of allocation frequency affairs Department Communication and Media Commission, Baghdad, Iraq. Email: A.majeed@cmc.iq

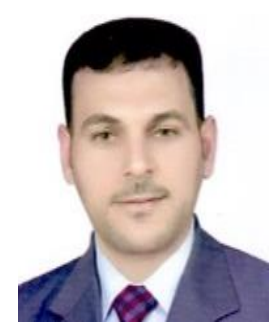

Mohammed Jawad Al_Dujaili AL_khazraji awarded B.S. degree in communication engineering from University of Al-Furat Al-Awsat Technical, Technical College of Engineering, Najaf, Iraq in 2008 and M.S. degree in communication system engineering from Ferdowsi university, Iran, in 2018. Currently, he is a member staff at the Department of Electronic and Communication, Faculty of Engineering, University of Kufa, Iraq. His research interest includes the development of Wireless communications and signal processing as well as image, speech processing and radar, 5G. Email: Mohammed.challab@uokufa.edu.iq 